\title{
Remission of In-Transit Melanoma following Combination BRAF and MEK Inhibition Therapy: A Case Report
}

\author{
S Caleb Freeman ${ }^{1}$, Edibaldo Silva ${ }^{2}$ and Christopher Huerter ${ }^{1}$ \\ ${ }^{1}$ Department of Dermatology, Creighton University School of Medicine, USA \\ ${ }^{2}$ Department of Surgical Oncology, University of Nebraska Medical Center, USA
}

\section{Introduction}

Satellite and in-transit melanoma represent the metastasis of cutaneous melanoma along lymphatics between the primary lesion and the regional lymph nodes. In-transit melanoma often presents as a reoccurrence of previously resected melanoma, and as such is associated with a poorer prognosis than primary melanoma [1]. Response rate to therapy is often suboptimal, with 5-year survival rates of $24-54 \%$ at 5 years [2]. Treatment protocols for in-transit melanoma are less standardized than many other clinical scenarios, but possibilities for treatment include surgical removal, palliative radiotherapy, isolated limb perfusion, intra-lesional T-VEC injections, systemic targeted therapy and immunotherapy [3]. This case presents a successful selective approach for the management of refractory in-transit melanoma that included treatment with combination BRAF inhibitor dabrafenib and MEK inhibitor trametinib for a patient with stage IV melanoma.

\section{Case Report}

A 58-year-old white woman was referred for a $2.5-\mathrm{cm}$ enlarging blackish brown patch on her left medial ankle that had recently begun bleeding. An excisional biopsy found malignant nodular melanoma with positive margins. She underwent wide re-excision and sentinel lymph node biopsy. Results showed residual malignant nodular melanoma with a Breslow depth of $1.1 \mathrm{~mm}$, Clark's level IV with negative margins. All 3 sentinel lymph nodes in the left groin were negative. She was followed clinically for 4 years, at which point she developed an extensive amount of new pigmented nodules on her left lower extremity (Figure 1). Biopsies were taken of a left pretibial nodule as well as a left lower lateral pretibial nodule. Both were consistent with malignant melanoma in-transit metastases that tested positive for a BRAF V600E mutation. In the absence of systemic metastases by PET scan, initial treatment consisted of heated isolated limb infusion with the alkylating agent melphalan $7.5 \mathrm{mg}$ per liter of volume, adjusted for patient's ideal body weight. While the patient initially showed improvement, with a near complete resolution of her in-transit leg disease for a period of 6 months, she later developed a painful pre-tibial mass on the leg which was treated with palliative radiation therapy. Unfortunately, and as often seen following in-transit disease, the patient developed progres- sive soft tissue metastases outside the perfused extremity, with new lesions appearing in the right shoulder and occiput. Based on the cross-over sensitivity of BRAF positive disease to systemic immunotherapy, she was treated with 4 cycles of pembrolizumab administered at $2 \mathrm{mg} / \mathrm{kg}$ every 2 weeks. Evidence of progression on this regimen led to a switch over to BRAF-targeted therapy with dabrafenib and trametinib. Patient received $150 \mathrm{mg}$ dabrafenib every 12 hours and $2 \mathrm{mg}$ trametinib once daily. As often seen, she showed complete resolution of nearly all disease within 4 weeks. Four months after completing treatment the skin and subcutaneous tissue remained clear (Figure 2). Patient remains on combination therapy to date.

\section{Discussion}

Combined dabrafenib and trametinib therapy is a potential therapeutic option for the treatment of BRAF-positive intransit melanoma metastases resistant to other therapies. This patient's disease was refractory to multiple traditional treatment regimens for in-transit melanoma, including isolated limb infusion and immune checkpoint therapy.

Both dabrafenib and trametinib were originally approved as monotherapy for BRAF V600 mutation-positive metastatic melanoma. In a phase III trial, therapy with dabrafenib, a BRAF inhibitor, showed a $50 \%$ response rate and progressionfree survival of 5.1 months [4].

Trametinib also targets the MAPK pathway by inhibiting MEK, a BRAF substrate. Median progression-free survival of trametinib in a phase III trial was 4.8 months with an overall survival of $81 \%$ [5]. However, a common mechanism of resistance to these treatments in monotherapy is the

*Corresponding author: S Caleb Freeman, Department of Dermatology, Creighton University School of Medicine, USA

Accepted: January 17, 2019

Published online: January 19, 2019

Citation: Freeman SC, Silva E, Huerter C (2019) Remission of In-Transit Melanoma following Combination BRAF and MEK Inhibition Therapy: A Case Report. Arch Surg Dermatol 2(1):1820

Copyright: (c) 2019 Freeman SC, et al. This is an open-access article distributed under the terms of the Creative Commons Attribution License, which permits unrestricted use, distribution, and reproduction in any medium, provided the original author and source are credited. 

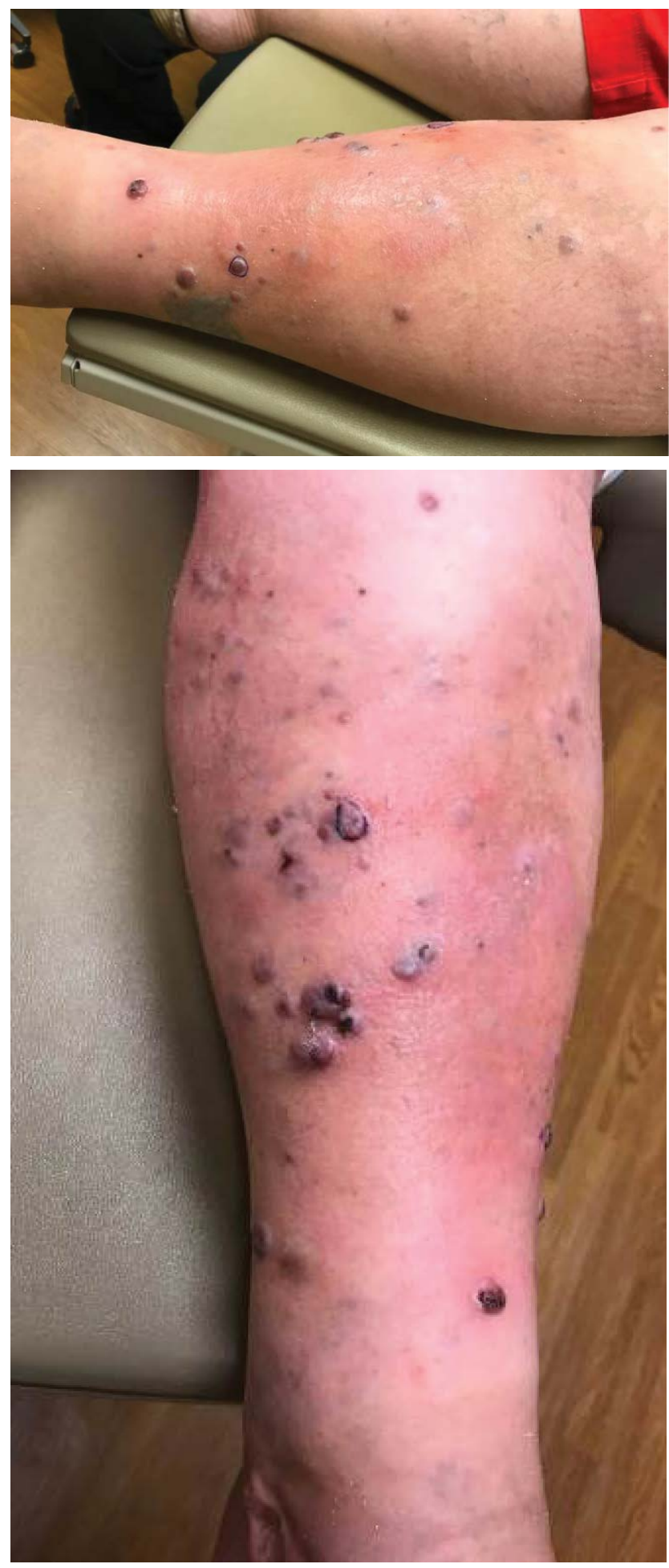

Figure 1: Melanoma in-transit metastases on the left leg.

development of bypass pathways such as MAPK reactivation [6]. Previous studies showed that resistance to monotherapy with a BRAF inhibitor such as dabrafenib developed within 6 to 7 months of initiating therapy in $50 \%$ of patients [7].

Because dabrafenib and trametinib both target the same pathway but via different mechanisms, combination therapy is now the preferred option to limit the development of resistance and thus improve outcomes [8]. The phase III clinical trial $\mathrm{COMBI}-\mathrm{d}$ compared therapy with both dabrafenib
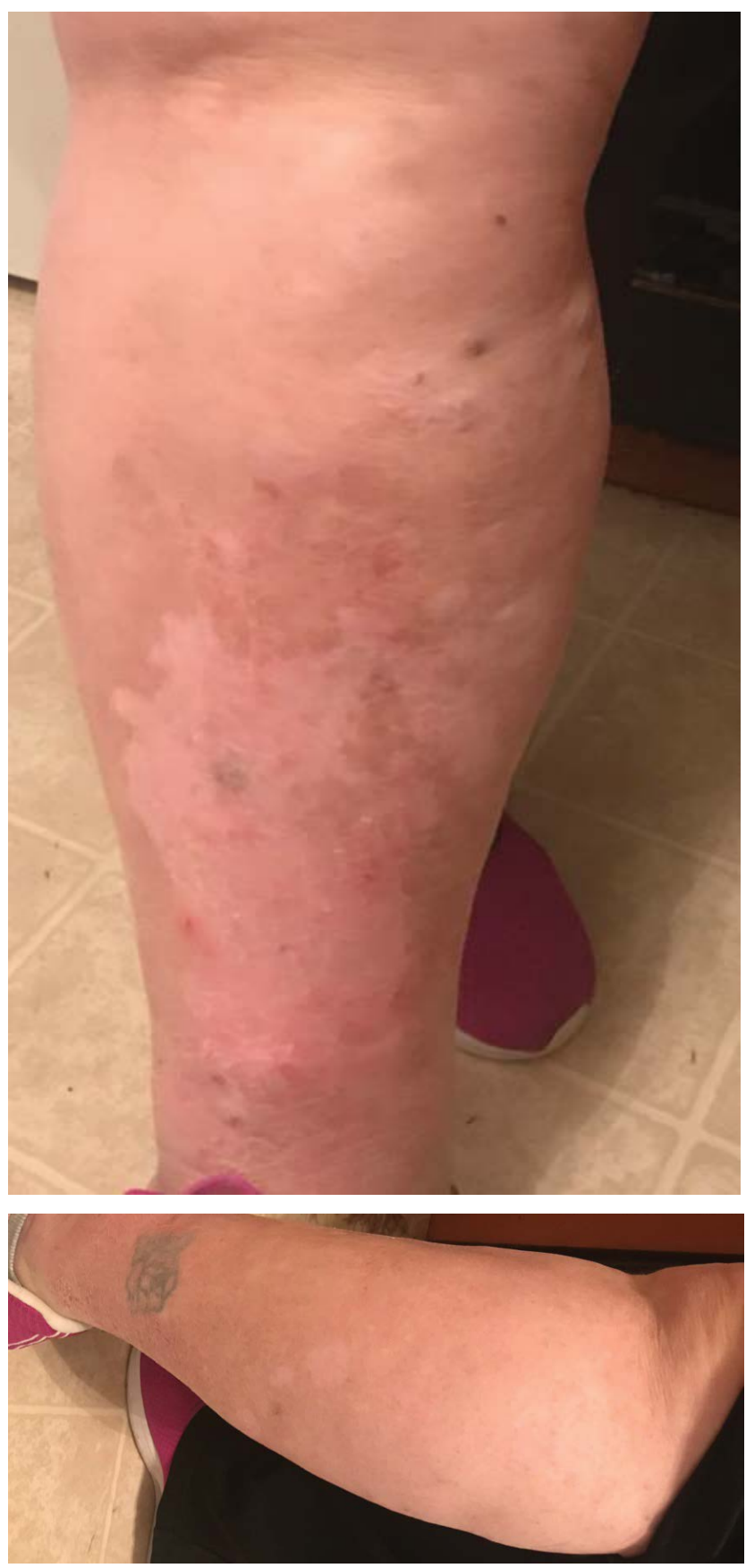

Figure 2: Patient's lower limb after treatment with the combination therapy.

and trametinib to dabrafenib alone. Results showed a $25 \%$ reduction in the risk of progression or death [9]. A separate study showed that combined therapy inhibiting both BRAF and MEK showed an improved response when compared to monotherapy, with $70 \%$ of patients experiencing a complete or partial response within a year [10].

This patient presently remains on combination therapy with dabrafenib and trametinib. Currently no guidelines for cessation of treatment exist, and physicians must balance the potential hazard of discontinuing combination treatment with the immense financial burden this treatment method places on both the patient and health care system. Further research and analysis is needed to guide physicians in determining 
a length of therapy for patients treated with combination therapy.

This case demonstrates the selective and orderly application of newly available but limited local and systemic options for the treatment of BRAF V600 mutation-positive intransit melanoma. Because treatment for in-transit melanoma is less standardized than other variants of melanoma, and in view of its predilection for subsequent systemic failure, the choice of available options may differ from individual to individual. The goal should be the complete resolution of disease while keeping in mind that distant metastases may lurk in the background for which there are limited options at hand should they become evident on follow up. In addition, the complete resolution of this patient's disease within 4 weeks reaffirms the efficacy of using combination therapy for in-transit melanoma with distant metastases.

\section{References}

1. Stucky CC, Gray RJ, Dueck AC, et al. (2010) Risk factors associated with local and in-transit recurrence of cutaneous melanoma. Am J Surg 200: 770-775.

2. Francken $A B$, Accortt NA, Shaw HM, et al. (2008) Prognosis and determinants of outcome following loco-regional or distant recurrence in patients with cutaneous melanoma. Ann Surg Oncol 15: 1476-1484.
3. Testori A, Ribero S, Bataille V (2017) Diagnosis and treatment of in-transit melanoma metastases. Eur J Surg Oncol 43: 544-560.

4. Hauschild A, Grob JJ, Demidov LV, et al. (2012) Dabrafenib in BRAF-mutated metastatic melanoma: A multicentre, open-label, phase 3 randomised controlled trial. Lancet 380: 358-365.

5. Flaherty KT, Robert C, Hersey P, et al. (2012) Improved survival with MEK inhibition in BRAF-mutated melanoma. $\mathrm{N}$ Engl J Med 367: 107-114.

6. Emery CM, Vijayendran KG, Zipser MC, et al. (2009) MEK1 mutations confer resistance to MEK and B-RAF inhibition. Proc Natl Acad Sci U S A 106: 20411-20416.

7. Sosman JA, Kim KB, Schuchter L, et al. (2012) Survival in BRAF V600-mutant advanced melanoma treated with vemurafenib. $\mathrm{N}$ Engl J Med 366: 707-714.

8. Heppt MV, Tietze JK, Graf SA, et al. (2015) Combination therapy of melanoma using kinase inhibitors. Curr Opin Oncol 27: 134-140.

9. Long GV, Stroyakovskiy D, Gogas H, et al. (2014) Combined BRAF and MEK inhibition versus BRAF inhibition alone in melanoma. $\mathrm{N}$ Engl J Med 371: 1877-1888.

10. KT Flaherty, JR Infante, A Daud, et al. (2012) Combined BRAF and MEK inhibition in melanoma with BRAF V600 mutations. N Engl J Med 367: 1694-1703. 